\title{
Incorporating radiomics into clinical trials: expert consensus endorsed by the European Society of Radiology on considerations for data-driven compared to biologically driven quantitative biomarkers
}

\author{
Laure Fournier $^{1,2,3}$ - Lena Costaridou ${ }^{2,4}$. Luc Bidaut ${ }^{3,5}$ - Nicolas Michoux ${ }^{3,6}$. Frederic E. Lecouvet ${ }^{3,6}$. \\ Lioe-Fee de Geus-Oei $^{3,7,8} \cdot$ Ronald Boellaard $^{2,9,10}$. Daniela E. Oprea-Lager ${ }^{3,9}$. Nancy A Obuchowski ${ }^{10,11}$. \\ Anna Caroli ${ }^{2,12}$. Wolfgang G. Kunz ${ }^{3,13}$ • Edwin H. Oei ${ }^{2,14}$ • James P. B. O'Connor ${ }^{2,15}$ - Marius E. Mayerhoefer ${ }^{2,16}$. \\ Manuela Franca ${ }^{2,17}$. Angel Alberich-Bayarri ${ }^{2,18}$. Christophe M. Deroose ${ }^{3,19,20} \cdot$ Christian Loewe $^{2,21}$. \\ Rashindra Manniesing ${ }^{2,22}$. Caroline Caramella ${ }^{3,23} \cdot$ Egesta Lopci ${ }^{3,24}$ - Nathalie Lassau ${ }^{2,3,10,25}$ - Anders Persson ${ }^{2,26}$. \\ Rik Achten ${ }^{2,27} \cdot$ Karen Rosendahl $^{2,28}$. Olivier Clement ${ }^{1,2} \cdot$ Elmar Kotter $^{2,29} \cdot$ Xavier Golay $^{2,10,30} \cdot$ Marion Smits $^{2,3,14}$. \\ Marc Dewey $^{2,31}$ - Daniel C. Sullivan ${ }^{2,10,32}$ - Aad van der Lugt ${ }^{2,14}$ - Nandita M. deSouza ${ }^{2,3,10,33}$ (D) European Society of \\ Radiology 34
}

Received: 14 July 2020 / Revised: 16 November 2020 / Accepted: 3 December 2020 / Published online: 25 January 2021

(C) The Author(s) 2021, corrected publication 2021

\begin{abstract}
Existing quantitative imaging biomarkers (QIBs) are associated with known biological tissue characteristics and follow a wellunderstood path of technical, biological and clinical validation before incorporation into clinical trials. In radiomics, novel datadriven processes extract numerous visually imperceptible statistical features from the imaging data with no a priori assumptions on their correlation with biological processes. The selection of relevant features (radiomic signature) and incorporation into clinical trials therefore requires additional considerations to ensure meaningful imaging endpoints. Also, the number of radiomic features tested means that power calculations would result in sample sizes impossible to achieve within clinical trials. This article examines how the process of standardising and validating data-driven imaging biomarkers differs from those based on biological associations. Radiomic signatures are best developed initially on datasets that represent diversity of acquisition protocols as well as diversity of disease and of normal findings, rather than within clinical trials with standardised and optimised protocols as this would risk the selection of radiomic features being linked to the imaging process rather than the pathology. Normalisation through discretisation and feature harmonisation are essential pre-processing steps. Biological correlation may be performed after the technical and clinical validity of a radiomic signature is established, but is not mandatory. Feature selection may be part of discovery within a radiomics-specific trial or represent exploratory endpoints within an established trial; a previously validated radiomic signature may even be used as a primary/secondary endpoint, particularly if associations are demonstrated with specific biological processes and pathways being targeted within clinical trials.

\section{Key Points}

- Data-driven processes like radiomics risk false discoveries due to high-dimensionality of the dataset compared to sample size, making adequate diversity of the data, cross-validation and external validation essential to mitigate the risks of spurious associations and overfitting.

- Use of radiomic signatures within clinical trials requires multistep standardisation of image acquisition, image analysis and data mining processes.

- Biological correlation may be established after clinical validation but is not mandatory.
\end{abstract}

Keywords Radiology $\cdot$ Statistics and numerical data $\cdot$ Standardization $\cdot$ Validation studies $\cdot$ Clinical trial

Nandita M. deSouza

nandita.deSouza@icr.ac.uk

Extended author information available on the last page of the article

\section{Abbreviations}

ADC Apparent diffusion coefficient

CE Conformite Europeenne 


$\begin{array}{ll}\text { CNN } & \text { Convolutional neural networks } \\ \text { CT } & \text { Computerised tomography } \\ \text { DL } & \text { Deep learning } \\ \text { DR } & \text { Deep radiomics } \\ \text { EGFR } & \text { Epidermal growth factor receptor } \\ \text { FDG } & \text { Fluorodeoxyglucose } \\ \text { IBSI } & \text { Image biomarker standardisation initiative } \\ \text { MeSH } & \text { Medical Subject Headings } \\ \text { MRI } & \text { Magnetic resonance imaging } \\ \text { PET } & \text { Positron emission tomography } \\ \text { QA } & \text { Quality assurance } \\ \text { QC } & \text { Quality control } \\ \text { QIBs } & \text { Quantitative imaging biomarkers } \\ \text { SPECT } & \text { Single photon emission computed tomography } \\ \text { SUV } & \text { Standardised uptake value } \\ \text { VOI } & \text { Volume of interest }\end{array}$

\section{Introduction}

Quantitative imaging biomarkers (QIBs) are associated with tissue characteristics that are altered by disease and its treatment. Necrosis decreases tissue cellularity and increases water content manifesting as an increase in T2 [1], a reduction in glucose uptake [2] and an increase in elasticity [3]. Perfusion imaging detects and characterises hypervascular lesions such as cancers, or monitors the effect of anti-angiogenic drugs [4, 5]. Implementation of QIBs into clinical trials follows a welldefined path from discovery, through a process of technical and biological validation, to implementation and clinical validation. A roadmap defining the process was published as a consensus statement from multiple stakeholders [6]. Despite this, QIBs have been slow to be adopted as trial endpoints because of the relative complexity of imaging protocols and variability of the quantified output under differing conditions (e.g. hardware, software, protocol and observer variability) [7].

Recently, a new approach to derive imaging biomarkers has been advocated through the concept of radiomics $[8,9]$. This data-driven framework 'discovers' quantitative information within images by extracting high-dimensional data ('features') beyond that visually perceptible, using computational statistics (often based on machine learning algorithms) to predict or establish association with a meaningful clinical endpoint $[10,11]$. Technical and clinical performance of the 'radiomic signature' (specific combination of mathematically derived features) determines its appropriateness. If considered necessary, a link to a biological process is explored a posteriori [12]. Radiomic signatures have been associated with outcome or response [13], and may be used together with clinical, histological and genomic metrics as part of a nomogram of features [14]. The exponential rise in publications involving data-driven biomarkers has not been accompanied by a mechanism-based understanding of their nature but focuses on their ability to classify disease and patient outcome (Fig. 1). Radiomics has been used for detecting cancer [15], cancer staging [16], performing classifications [17], assessing response to chemotherapy [18], radiation therapies [19-22], immunotherapy [23-26] and predicting/prognosing survival [27].

A major disadvantage of a non-mechanistic data-driven approach is that random chance associations may occur. Most studies look at the associations between a large number of features extracted from discretised images and prognosis/
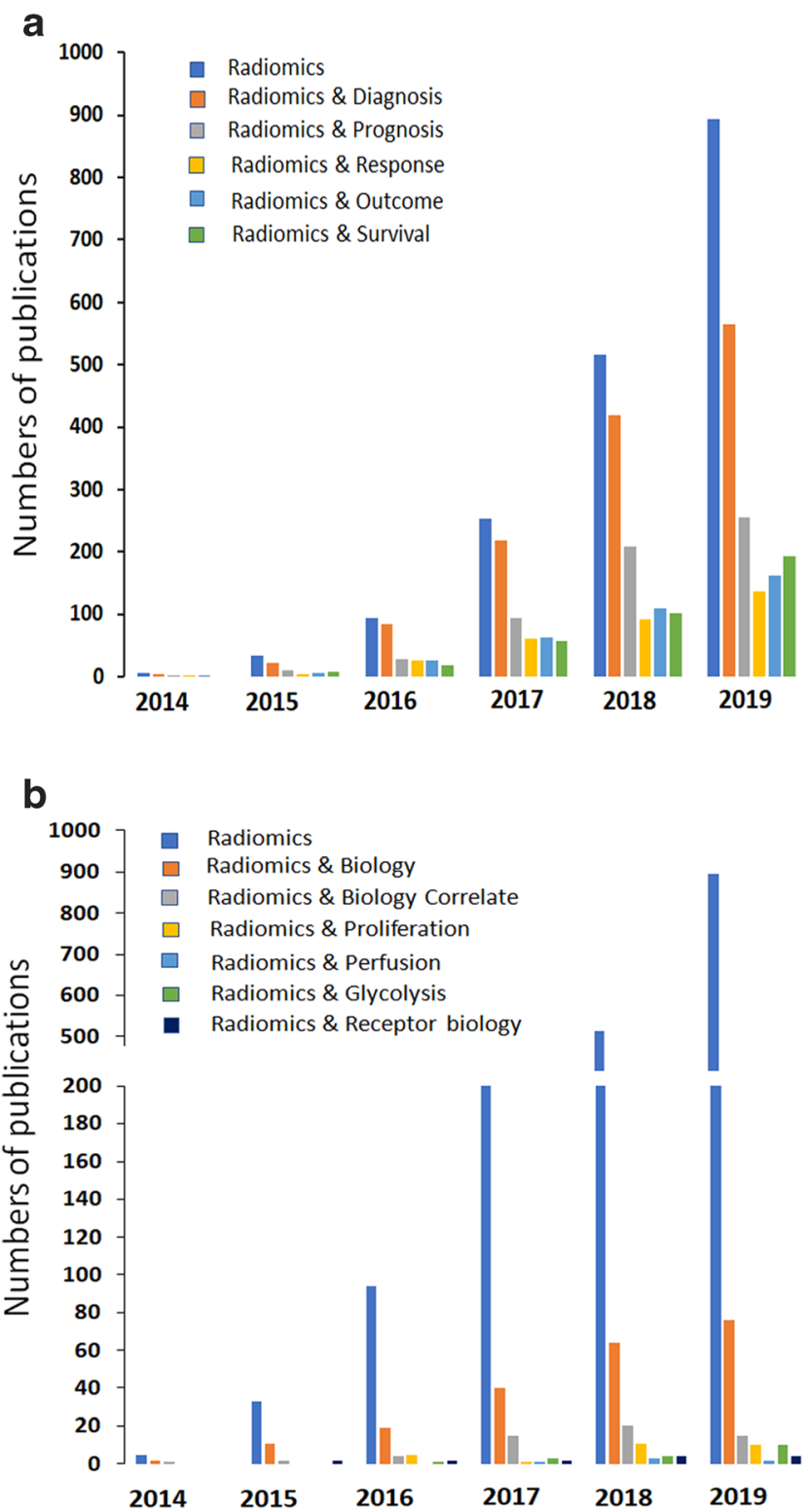

Fig. 1 Increase in radiomics related publications over last 6 years (a) by patient status/outcome and (b) by biological association using data extracted from PubMed using the indicated MeSH terms. The exponential increase in radiomics publications relates mainly to usage as indicated in $\mathbf{a}$, and not to their underlying biological associations as indicated in $\mathbf{b}$ 
response/outcome in an inadequate number of samples. For biomarker profiles that rely on statistical rather than biological associations, generalisation and scalability to multicentre trials requires more than a simple standardisation process. Also, their validation pathway needs to incorporate measures that may differ substantially from traditionally accepted methods. This article prepared by imaging experts from the European Society of Radiology EIBALL (European Imaging Biomarker ALLiance) and the EORTC (European Organisation for Research and Treatment of Cancer) Imaging Group with representatives from QIBA (Quantitative Imaging Biomarkers Alliance) examines how the process of standardising and validating data-driven imaging biomarkers differs from those based on biological associations, and what measures need to be considered when implementing them into clinical trials and, eventually, into clinical routine. Structured discussions were conducted via teleconferencing and written communications.

\section{Standardising the radiomics process for clinical trials}

Radiomics analyses rely on image acquisition, image analysis and computational statistics [28], so standardisation of these domains is mandatory prior to their validation (Table 1). As radiomics analyses have been applied to CT [29-31], MRI
[32-36], nuclear medicine using FDG-PET [37-42] and other tracers [43, 44], and ultrasound [45], image acquisition standardisation needs to consider modality, scanner and scan protocol. Standardisation of image analysis needs to consider software (consistency of technical implementation) and subjectivity (human interaction). Standardisation of computational statistics needs to consider adequacy, performance and requirements for validation of algorithms and models (Fig. 2).

Image acquisition and normalisation An element of diversity of acquisition protocols or machines is advantageous at the discovery phase of data-driven biomarkers so that the identified radiomic signatures used in clinical trials are robust enough across a range of platforms [46]. Datasets utilised for radiomic signature development must be representative of the disease and capture the variability and severity for which they will be used. Within a clinical trials framework, as with previously published recommendations and guidelines $[6$, 47-49], an optimised tightly controlled standardised imaging protocol ensures image quality (low level of noise, artifactfree, spatial resolution) and stability over time, with known intra- and inter-site reproducibility that does not exceed the expected level of change associated with the trial intervention [50]. Phantom studies are limited for quality control of highdimensionality information [51] because a suitable phantom would need to exhibit high-dimensionality in a realistic setting and cover the requirements of each type of feature.

Table 1 Comparison of standardisation steps for biologically driven and data-driven biomarkers ( $Q A$, quality assurance; $Q C$, quality Control; VOI, volume of interest)

\begin{tabular}{|c|c|c|}
\hline Steps & Biologically driven quantitative biomarkers & Data-driven quantitative biomarkers \\
\hline Image acquisition & $\begin{array}{l}\text { - Standardised protocols (single and multicentre) } \\
\text { - QA/QC process across instruments, sites } \\
\text { - Stability of measurement monitored with phantom } \\
\text { studies; may be strengthened by human subject } \\
\text { test-retest }\end{array}$ & $\begin{array}{l}\text { - Non-standardised protocols in discovery phase } \\
\text { followed by standardised protocols within trials } \\
\text { - QA/QC process across instruments, sites } \\
\text { - Stability of measurement requires human } \\
\text { subject test-retest }\end{array}$ \\
\hline VOI delineation & $\begin{array}{l}\text { - Can be manual or semi-automated } \\
\text { - Can be machine-learnt } \\
\text { - Deep learning available but infrequently used }\end{array}$ & $\begin{array}{l}\text { - Can be manual or semi-automated } \\
\text { - Can be machine-learnt } \\
\text { - Can be derived from fully convolutional neural } \\
\text { networks }\end{array}$ \\
\hline Data analysis & $\begin{array}{l}\text { - Commercial or academic software applicable to } \\
\text { datasets } \\
\text { regardless of their source }\end{array}$ & $\begin{array}{l}\text { - Algorithms used are specific to image datasets } \\
\text { and may require adaptation and standardisation } \\
\text { for individual situations or new datasets* }\end{array}$ \\
\hline Biomarker extraction & $\begin{array}{l}\text { - Follows standard formula that describes the } \\
\text { biological feature (e.g. tissue density, perfusion, } \\
\text { diffusion, standardised uptake of radiotracers } \\
\text { related to a biological process/receptor status) }\end{array}$ & $\begin{array}{l}\text { - Algorithm-based mathematical feature extraction } \\
\text { not directly linked to a biological process, followed } \\
\text { by selection of feature combination that best separate } \\
\text { disease from no disease, good from poor outcome } \\
\text { (e.g. shape features such as diameter, sphericity; } \\
\text { histogram-derived } \\
\text { features such as median, skewness, } \\
\text { entropy; texture features such as contrast, } \\
\text { homogeneity, Haralick variance) }\end{array}$ \\
\hline Biomarker interpretation & - Directly linked to biological process & - Indirect associations with biological process assumed \\
\hline
\end{tabular}

*https://ibsi.readthedocs.io/en/latest/; *https://www.lifexsoft.org/; *http://www.eletel.p.lodz.pl/programy/mazda/; *https://nmmitools.org/2019/01/01/pyradiomics/ 


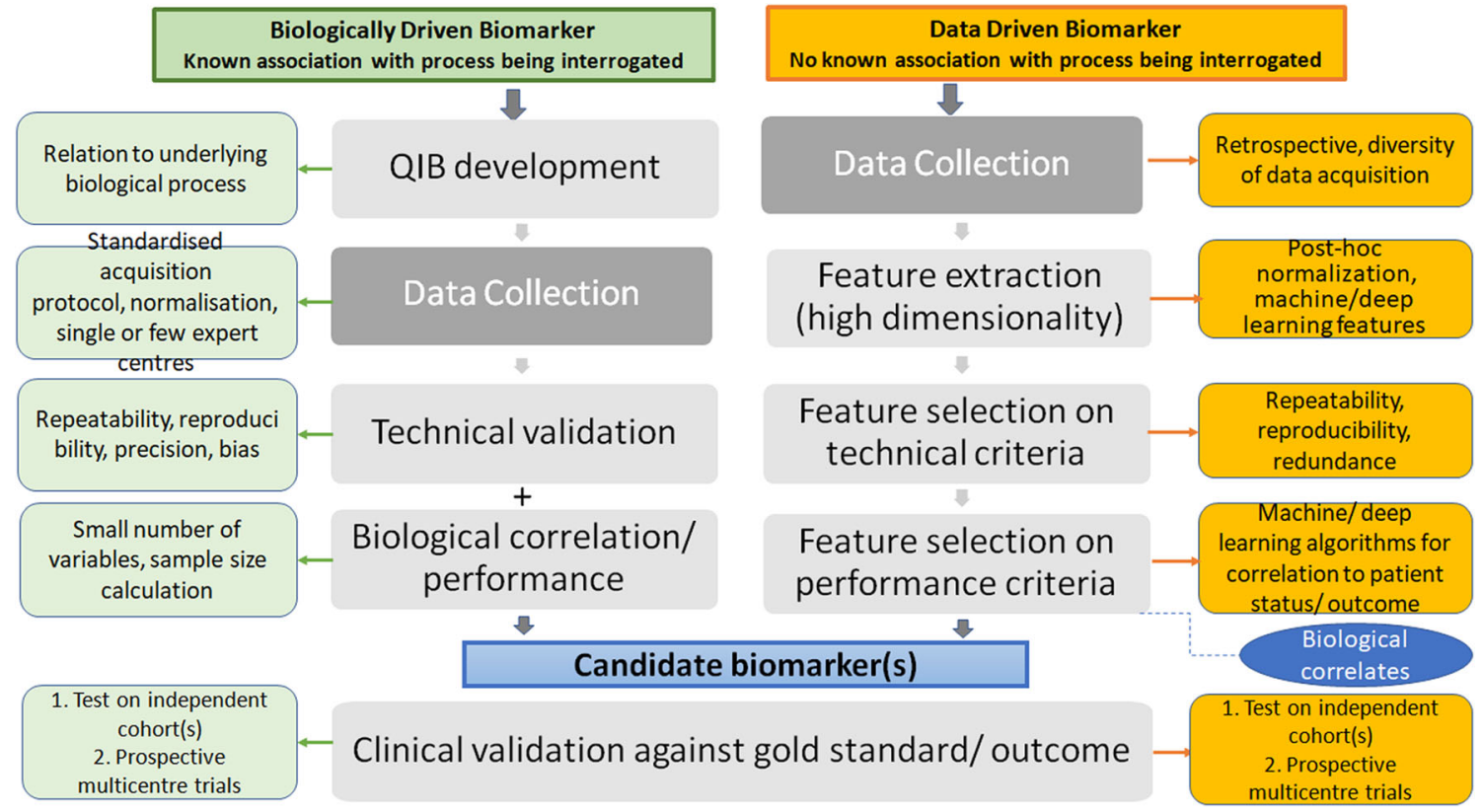

Fig. 2 Pathways comparing processes required for biologically driven and data-driven biomarkers. Biologically driven biomarkers derived from known associations with a specific biological process require a specific predetermined acquisition protocol and image processing technique and involve technical, biological and clinical validation steps with recognised requirements (green boxes). Data-driven biomarkers assume that the statistical features that relate to the biological process or outcome are unknown so that all possible features are extracted from the images and steps to determine their technical and clinical performance are needed (orange boxes). Feature extraction and selection depend on the data mining process (machine and deep learning algorithms). A training dataset and validation dataset allow selection of most promising feature(s), and an independent test dataset allows evaluation of performance of imaging biomarker. Biological links are explored a posteriori

Image analysis-segmentation As with biologically driven biomarkers, manual region of interest delineation introduces inter- and intra-observer variability because of variation in border perception. Observer training and working to protocol assists in this regard. Semi-automated segmentation methods, e.g. region-growing or level set active contour models [62] and deep learning methods [63], are more reproducible [64], but they are dependent on their training set, which may introduce other errors. Quantitative verification metrics [65], such as Dice coefficient, and Hausdorff distance metrics, help determine segmentation reproducibility. Images that require alignment for different time series data, parametric maps and modalities should evaluate deviations in locations (distance) of pairs of homologous landmark points, especially important for non-rigid image registration $[66,67]$.

Image analysis-feature extraction 'Hand-crafted' radiomics extracts predefined human-engineered features from the volume-of-interest (VOI) [17]. These include shape characteristics, intensity histogram metrics and texture parameters (local binary patterns, grey-level co-occurrence, run-length, zone-length and neighbourhood different matrices, auto-regressive model, Markov random fields, Riesz wavelets, S-transform, fractals) which require specific assumptions in their computation, so that software 
implementations on different platforms (even if all are IBSI compliant) and between different versions of the same software can lead to different results [68]. Recommendations on calculating and reporting radiomic features have been proposed, and both mathematical equations and pre-processing applied should be reported. The information and framework provided through IBSI [61] should also be followed as much as possible to ensure the quality and relevance of the post-processing (denoising, resampling, enhancement, spatial alignment correction, segmentation and feature extraction). Other descriptive (radiologist-scored), functional (SUV, ADC, $\mathrm{K}^{\text {trans }}$ ) or clinical parameters may be added to the radiomic signature if pertinent.

Computational statistics - feature selection Several tools are described [69-72]. To identify relevant, non-redundant and stable features with which to build models, three categories of technique are employed. Filter methods (ANOVA, correlation, RELIEF [73]) rely on a criterion function, have low computational cost and are less prone to overfitting, by separating selection from model building; however, they are more unstable to different datasets. Wrapper methods (forward selection, backward elimination, stepwise selection) incorporate a specific machine learning algorithm to eliminate features but have increased computational cost and high probability of overfitting, since model training uses feature combinations that include common features. Embedded methods (LASSO, RIDGE regression) embed features successively and penalise the coefficients of a model that contribute to overfitting at each iteration. They represent a trade-off between filter and wrapper methods.

Computational statistics-classifier/model After dimension reduction, selected features are investigated for their association with clinical outcome using tools such as univariable or multivariable logistic regression, decision tree, random forest, support vector machine, neural networks, all described extensively in previous publications [65-68] and used for QIBs and radiomic analyses [24]. Classifiers are differentiated depending on the nature of the clinical outcome, i.e. discrete (mainly binary) or continuous $[74,75]$. No tool has proved universally superior and most require a compromise between complexity of tuning versus interpretability of results.

Computational statistics-deep radiomics (DR) A recent evolution has been the integration of radiomics with deep learning (DL) [76-78]. 'Discovery Radiomics' automatically extracts deep features relevant to a given query (e.g. diagnosis, prognosis) from the data, and the resulting trained model can be applied to complete datasets, avoiding the errorprone segmentation step. As DL can include multiple data types, relevant information in electronic patient records can be exploited.

\section{Validating the radiomics output}

Technical validation Following identification of a radiomics signature associated with disease/outcome, two fully independent datasets are needed, one for training and cross-validation (internal validation), and at least one other to test the final model and confirm generalisability and performance (external validation). Both training and testing datasets should be of sufficient uniform quality (data balancing) and representative for the patient population for which the radiomics model is intended. An adequate sample (size and diversity) is essential for the training and validation datasets, with respect to the number and type of features ('signature') considered. Testing the model with a dataset containing a different prevalence of cases and/or a high degree of imbalance may result in overoptimistic conclusions. Feature selection avoids overparameterised models, reduces dimensionality of the feature space (data dimension reduction) and ensures that only a small and stable subset of original features relevant to the task are retained. A strategy to cross-validate the structure of the model requires careful considerations regarding sample size, accuracy estimation and the choice of the validation method (holdout, k-fold cross-validation, bootstrap). Grid searches pose the danger of overfitting, leading to overoptimistic model performance that is not reproduced on other datasets or in clinical practice. Finally, repeatability and reproducibility of the signature in a multicentre context (affected by imaging apparatus, acquisition protocols and analysis methods) is a crucial step in technical validation [79-81]. As with QIBs, radiomics models should be tested with cross-institutional clinical training and testing datasets to guarantee generalisability to representative patient populations.

Biological validation Biological correlation with liquid/tissue biopsies may be performed after the technical and clinical validity of a radiomic signature is established but is not mandatory. A radiomic signature that is related to survival outcomes may potentially reflect a tissue phenotype associated with a specific biology. Biological validation reduces the likelihood that radiomic features are selected by statistical chance or may be attributed to the nature of the data sample used for model development. It also offers the opportunity to reduce the number of selected features.

Clinical validation The process by which the clinical utility of a single quantitative feature, or multiple features embedded in a statistical model is demonstrated, allowing improvement of health outcomes (improved diagnosis or therapeutic management of a disease or individual patient) is being addressed slowly for radiomics. Following initial 'discovery', new and independent datasets are required to replicate the performance of the identified model and validate it clinically. Performance metrics, e.g. sensitivity and specificity, should be evaluated 
ideally in prospective trials, or prospectively in the clinic using routinely obtained clinical data (real-life conditions) in order to avoid bias. Table 2 lists some exemplar studies and their clinical use. Broadly speaking, standard recommendations for clinical validation and clinical utility assessment of any QIB should be followed and applied.

\section{Biological correlates of radiomic features}

Images provide an averaged macroscopic view (with large partial volume effects, both in space and time) of the geometry and/or function of the tissue. Radiomic features are statistical descriptors characterising the macroscopic visual aspect of images and only indirectly relate to the microscopic histological characteristics of the imaged tissue. Such features are then used as a statistical/phenomenological description of the outcome, and not embedded into an actual biological/physical model of this outcome that would unambiguously establish causality between features and outcome.

Radiomic information on visually imperceptible phenotypic characteristics such as intensity, shape, size and texture distinguish benign and malignant tumours, likely reflecting different cellular morphology [101]. In cervix cancer, radiomic features of low-volume tumours with radiomic profiles similar to high-volume tumours had a worse prognosis implying a more aggressive phenotype at an earlier stage [36]. In a lung cancer study, texture entropy and cluster features, as well as voxel intensity variance features, were associated with the immune system, the $\mathrm{p} 53$ pathway, pathways involved in cell cycle regulation [102] and for predicting EGFR mutation status [103]. Nevertheless, why specific features are associated with specific pathways remains unexplored and the relationship between radiomic signature and cell morphology, density, distribution pattern, alignment and organelle composition need further elucidation.

Although it is possible to extract mathematically hundreds or thousands of radiomic features from digital images, most studies to date suggest that less than 20 are indicative of unfavourable biology, and these largely relate to shape and textural uniformity. 2D shape features indicate more rapidly progressive disease with reduced overall survival in glioblastoma multiforme [104]. Shape and textural features from CT scans of lung cancer have been shown to predict unfavourable biology (nodal and distant metastases respectively) [105]. In

Table 2 Exemplar radiomics signature studies and their clinical use

\begin{tabular}{|c|c|c|c|c|}
\hline Radiomic analysis & Radiomic feature (process) & Modality & Tissue types investigated & Decision-making role \\
\hline Second-order statistics & Textural (Haralick, Gabor) & $\begin{array}{l}\text { CT [29-31] } \\
\text { MRI [24-26] } \\
\text { PET/CT } \\
\quad[37-42]\end{array}$ & $\begin{array}{l}\text { Lung, breast, brain, } \\
\text { liver, prostate, } \\
\text { head and neck, } \\
\text { lymph node, cervix }\end{array}$ & $\begin{array}{l}\text { - Prognostic } \\
\text { - Predictive } \\
\text { - Response } \\
\text { - Survival } \\
\text { - EGFR expression } \\
\text { - p53 mutation status }\end{array}$ \\
\hline \multirow[t]{2}{*}{$\begin{array}{l}\text { Higher-order } \\
\text { statistics } \\
\text { Filter grids extract } \\
\text { repetitive or } \\
\text { non-repetitive } \\
\text { patterns }\end{array}$} & Wavelets & $\begin{array}{l}\text { CT [82-87] } \\
\text { MRI [88-90] } \\
\text { PET/CT [91, } \\
\quad \text { 92] }\end{array}$ & $\begin{array}{l}\text { Lung, oesophagus, } \\
\text { brain, pancreas, } \\
\text { breast, head and } \\
\text { neck }\end{array}$ & $\begin{array}{l}\text { - Diagnostic } \\
\text { - Prognostic } \\
\text { - Predictive } \\
\text { - Response } \\
\text { - Survival } \\
\text { - Surgical resection margins }\end{array}$ \\
\hline & $\begin{array}{l}\text { Laplacian transforms } \\
\text { (bandpass filters) } \\
\text { Minkowski functions } \\
\text { (patterns of voxels with } \\
\text { intensity above threshold) } \\
\text { Fractal dimensions } \\
\text { (patterns imposed on } \\
\text { image and number of } \\
\text { grid elements containing } \\
\text { voxels of a specified value is } \\
\text { computed) }\end{array}$ & $\begin{array}{l}\text { CT }[93,94] \\
\text { MRI }[95-97] \\
\text { PET/CT }[92]\end{array}$ & $\begin{array}{l}\text { Brain, lung, rectum, cervix, } \\
\text { kidney }\end{array}$ & $\begin{array}{l}\text { Prognostic } \\
\text { Response }\end{array}$ \\
\hline Delta radiomics & Change in radiomic features & $\begin{array}{l}\mathrm{PET} / \mathrm{CT}[98, \\
99]\end{array}$ & Lung & Response \\
\hline $\begin{array}{l}\text { Dynamic radiomic } \\
\text { studies }\end{array}$ & Pharmacokinetic radiomic features & $\mathrm{PET} / \mathrm{CT}[100]$ & Lung & $\begin{array}{l}\text { Response, data highly } \\
\text { correlated to data from static } \\
\text { studies }\end{array}$ \\
\hline
\end{tabular}


prostate cancer, Gabor textural features (defining spatial frequency patterns within the image) were predictive of Gleason grade on MRI. As gland lumen shape features relate to Gleason grade, discriminability of Gabor features is a likely consequence of variations in gland shape and morphology at the tissue level [106]. In future, prospective selection of a handful of relevant features should become possible to interrogate specific biological processes and pathways being manipulated within clinical trials so that it may be possible for the clinical question to drive the choice of biomarker usage and analysis. However, understanding the biological basis for a biomarker to facilitate its acceptance into clinical practice is not the primary objective of a data-driven process such as radiomics. It may well be that reliable modelling of the outcome with a relatively high and clinically acceptable performance means that biological validation would not be a primary concern [107].

\section{Limitations of data-driven processes}

When defining training datasets for radiomic feature extraction and selection in clinical trials, case-control data may be considered but may underrepresent the disease. Enrichment of training datasets with normal and abnormal cases of varying disease severity is mandatory to achieve appropriate balance. Bias in the training datasets limits generalisability. For example, a radiomic signature developed on lung nodules detected on chest $\mathrm{x}$-rays in a population with a high prevalence of tuberculosis and few cancers will overdiagnose tuberculosis in a population with a high prevalence of cancer. Image acquisition bias (cases recognised as disease acquired with a specific protocol or device) where selected features are linked to image acquisition rather than to image content may fail to predict disease when applied to an independent population. Manual VOI segmentation and use of locally developed methodology risks discovery of features that are not generalisable and may be influenced by hardware or software-related factors rather than the disease itself. Diverse but balanced image acquisition conditions in the training dataset should counteract these effects. Though balance and diversity are necessary at the discovery stage, it is crucial to evaluate performance only on populations representative of the natural prevalence.

The radiomic process, which tests combinations of hundreds and thousands of parameters, risks false discovery. Traditional statistical corrections for multiple tests would lead to $p$ values impossible to reach. Strategies to reduce spurious correlations and overfitting include artificially increasing the number of samples by data augmentation (datasets flipped, rotated and deformed to simulate new patients). Crossvalidation or bootstrapping are alternative strategies, but an independent dataset to confirm the findings is always required.

\section{Implementation of radiomics in clinical trials}

Although the discovery phase requires image acquisition diversity, standardised protocols, pre- and post-processing methods, tools and algorithms for feature extraction are needed for incorporating into clinical trials and facilitated by centralised data analyses and publicly available analysis software (Table 3). To incorporate radiomics in clinical trials, three potential scenarios can be considered. Firstly, where radiomic signature discovery is the objective, a trial should follow the steps described and illustrated (Fig. 2). Secondly, a radiomic 'exploratory end-point' may form an ancillary
Table 3 Recommended process for inclusion of data-driven biomarkers into clinical trials

\begin{tabular}{|c|c|}
\hline Step & Recommended process for clinical trial inclusion \\
\hline Image acquisition & $\begin{array}{l}\text { Standardised protocol agreed with site with vendor-specific amendments } \\
\text { (incl. software version control) to achieve reproducibility of other QIBs } \\
\text { within accepted published standards }\end{array}$ \\
\hline Image acquisition — normalisation & Raw data saved. Image normalisation predefined \\
\hline Image analysis-segmentation & $\begin{array}{l}\text { If manual or semi-automated, done by centralised/core laboratory by }>1 \\
\text { observer to establish reproducibility. If automated, can be done with } \\
\text { CE-marked software with established limits of agreement at local sites }\end{array}$ \\
\hline Image analysis - feature extraction & $\begin{array}{l}\text { Use of validated features with established error margins, adapted for } \\
\text { individual situations. Discard redundant features. Test reproducibility, } \\
\text { repeatability within trial setting }\end{array}$ \\
\hline $\begin{array}{l}\text { Computational statistics - feature } \\
\text { and model selection }\end{array}$ & $\begin{array}{l}\text { Based on performance by association with trial endpoint (e.g. } \\
\text { response/survival) }\end{array}$ \\
\hline Validation & $\begin{array}{l}\text { Adequate sample size, test data on samples with similar characteristics, } \\
\text { cross-validation strategies, avoid over-fitted models }\end{array}$ \\
\hline Biomarker interpretation & Association with positive diagnosis, prognosis or outcome \\
\hline
\end{tabular}


study within an established trial. Here, a two-phase process would involve an initial phase utilising more than two-thirds of the final cohort data (training cohort) to identify the most promising feature(s) and a subsequent phase using the remaining patients (independent cohort) to evaluate the performance of the identified radiomic signature. Thirdly, where a previously validated radiomic signature is used, this could be incorporated into a clinical trial as a primary or secondary endpoint. In this last case, the pathway of a data-driven biomarker does not differ from a QIB.

\section{Summary and future perspective}

Data-driven imaging biomarkers provide information beyond that perceived by human readers. Their benefits may be exploited if specific standardisation and validation pathways are defined and the different/additional hurdles compared to more traditional QIBs are addressed. Effects of different types of processing on subsequent extracted feature variability and predictive model performance is an open area of research [13]. Availability of public access patient cohorts with welldocumented image datasets is expected to facilitate consensus regarding pre- and post-processing methods and determine utility of radiomics within clinical trials.

While radiomics may eventually encompass all quantitative image-derived information into a common framework, current implementations mostly relate to intensity, shape and textural features within a VOI. In the future, quantitative (or even qualitative) functional information, e.g. derived from PET, SPECT, pharmacokinetic modelling and other parametric imaging modalities, may form part of the radiomic signature, and require a smaller or biologically more meaningful set of parameters. Deep radiomics may also be deployed in trials, and recent studies have already demonstrated the potential of such approaches [108-111].

Regardless of definitive biological correlation, once adopted and properly deployed, data-driven biomarkers may be combined with clinical data and other biomarkers (biochemical, genetic, epigenetic, transcription factors, proteins). Such expanded use of radiomics should eventually improve disease characterisation, prognostic stratification and response prediction in clinical trials, ultimately advancing precision medicine.

Acknowledgements This paper was endorsed by the ESR Executive Council in December 2020.

Funding The authors state that this work has not received any funding.

\section{Compliance with ethical standards}

Guarantor Nandita M deSouza.

\section{Conflict of interest}

LF - Speaker fees from Sanofi, Novartis, Jannssen, General Electric.
Congress sponsorship from Guerbet. Industrial grant on radiomics from Invectys, Novartis. Co-investigator in grant with Philips, Ariana Pharma, Evolucare.

CC - personal fees from Pfizer, BMS, MSD, Roche and advisory role for Astra Zeneca.

CMD - Consulting or advisory roles with Ipsen, Novartis, Terumo, and Advanced Accelerator Applications; participation in speakers' bureaus with Terumo and Advanced Accelerator Applications; and travel, accommodations, or expenses with General Electric and Terumo.

XG: CEO of Gold Standard Phantoms, a company designing calibration devices for quantitative MRI.

All other authors- none.

Statistics and biometry No complex statistical methods were necessary for this paper.

Informed consent Not applicable in this perspectives paper.

Ethical approval Not applicable in this special report.

\section{Methodology}

- Special report

Open Access This article is licensed under a Creative Commons Attribution 4.0 International License, which permits use, sharing, adaptation, distribution and reproduction in any medium or format, as long as you give appropriate credit to the original author(s) and the source, provide a link to the Creative Commons licence, and indicate if changes were made. The images or other third party material in this article are included in the article's Creative Commons licence, unless indicated otherwise in a credit line to the material. If material is not included in the article's Creative Commons licence and your intended use is not permitted by statutory regulation or exceeds the permitted use, you will need to obtain permission directly from the copyright holder. To view a copy of this licence, visit http://creativecommons.org/licenses/by/4.0/.

\section{References}

1. Santamaria G, Velasco M, Bargallo X, Caparros X, Farrus B, Luis Fernandez P (2010) Radiologic and pathologic findings in breast tumors with high signal intensity on T2-weighted MR images. Radiographics 30:533-548

2. Parghane RV, Basu S (2020) PET/computed tomography in treatment response assessment in cancer: an overview with emphasis on the evolving role in response evaluation to immunotherapy and radiation therapy. PET Clin 15:101-123

3. Lee SH, Moon WK, Cho N et al (2014) Shear-wave elastographic features of breast cancers: comparison with mechanical elasticity and histopathologic characteristics. Invest Radiol 49:147-155

4. de Bazelaire C, Calmon R, Chapellier M, Pluvinage A, Frija J, de Kerviler E (2010) CT and MRI imaging in tumoral angiogenesis. Bull Cancer 97:79-90

5. Ammari S, Thiam R, Cuenod CA et al (2014) Radiological evaluation of response to treatment: application to metastatic renal cancers receiving anti-angiogenic treatment. Diagn Interv Imaging 95:527-539

6. O'Connor JP, Aboagye EO, Adams JE et al (2017) Imaging biomarker roadmap for cancer studies. Nat Rev Clin Oncol 14:169186

7. deSouza NM, Achten E, Alberich-Bayarri A et al (2019) Validated imaging biomarkers as decision-making tools in clinical 
trials and routine practice: current status and recommendations from the EIBALL* subcommittee of the European Society of Radiology (ESR). Insights Imaging 10:87

8. Gillies RJ, Kinahan PE, Hricak H (2016) Radiomics: images are more than pictures, they are data. Radiology 278:563-577

9. Pinto Dos Santos D, Dietzel M, Baessler B (2020) A decade of radiomics research: are images really data or just patterns in the noise? Eur Radiol. https://doi.org/10.1007/s00330-020-07108-w

10. Lambin P, Leijenaar RTH, Deist TM et al (2017) Radiomics: the bridge between medical imaging and personalized medicine. Nat Rev Clin Oncol 14:749-762

11. van Timmeren JE, Cester D, Tanadini-Lang S, Alkadhi H, Baessler B (2020) Radiomics in medical imaging-"how-to" guide and critical reflection. Insights Imaging 11:91

12. Sanduleanu S, Woodruff HC, de Jong EEC et al (2018) Tracking tumor biology with radiomics: a systematic review utilizing a radiomics quality score. Radiother Oncol 127:349-360

13. Nie K, Al-Hallaq H, Li XA et al (2019) NCTN assessment on current applications of radiomics in oncology. Int J Radiat Oncol Biol Phys 104:302-315

14. Wang T, Gao T, Yang J et al (2019) Preoperative prediction of pelvic lymph nodes metastasis in early-stage cervical cancer using radiomics nomogram developed based on T2-weighted MRI and diffusion-weighted imaging. Eur J Radiol 114:128-135

15. Cameron A, Khalvati F, Haider MA, Wong A (2016) MAPS: a quantitative radiomics approach for prostate cancer detection. IEEE Trans Biomed Eng 63:1145-1156

16. Ma X, Shen F, Jia Y, Xia Y, Li Q, Lu J (2019) MRI-based radiomics of rectal cancer: preoperative assessment of the pathological features. BMC Med Imaging 19:86

17. Yun J, Park JE, Lee H, Ham S, Kim N, Kim HS (2019) Radiomic features and multilayer perceptron network classifier: a robust MRI classification strategy for distinguishing glioblastoma from primary central nervous system lymphoma. Sci Rep 9:5746

18. Shi L, He Y, Yuan Z et al (2018) Radiomics for response and outcome assessment for non-small cell lung cancer. Technol Cancer Res Treat 17:1533033818782788

19. Peeken JC, Bernhofer M, Wiestler B et al (2018) Radiomics in radiooncology - challenging the medical physicist. Phys Med 48: $27-36$

20. Reuze S, Schernberg A, Orlhac F et al (2018) Radiomics in nuclear medicine applied to radiation therapy: methods, pitfalls, and challenges. Int J Radiat Oncol Biol Phys 102:1117-1142

21. Elhalawani H, Lin TA, Volpe S et al (2018) Machine learning applications in head and neck radiation oncology: lessons from open-source radiomics challenges. Front Oncol 8:294

22. Bibault JE, Xing L, Giraud P et al (2020) Radiomics: a primer for the radiation oncologist. Cancer Radiother. https://doi.org/10. 1016/j.canrad.2020.01.011

23. El Naqa I, Ten Haken RK (2018) Can radiomics personalise immunotherapy? Lancet Oncol 19:1138-1139

24. Sun R, Limkin EJ, Vakalopoulou M et al (2018) A radiomics approach to assess tumour-infiltrating CD8 cells and response to anti-PD-1 or anti-PD-L1 immunotherapy: an imaging biomarker, retrospective multicohort study. Lancet Oncol 19:1180-1191

25. Trebeschi S, Drago SG, Birkbak NJ et al (2019) Predicting response to cancer immunotherapy using noninvasive radiomic biomarkers. Ann Oncol 30:998-1004

26. Basler L, Gabrys HS, Hogan SA et al (2020) Radiomics, tumor volume and blood biomarkers for early prediction of pseudoprogression in metastatic melanoma patients treated with immune checkpoint inhibition. Clin Cancer Res. https://oi.org/ 10.1158/1078-0432.CCR-20-0020

27. Choe J, Lee SM, Do KH et al (2020) Outcome prediction in resectable lung adenocarcinoma patients: value of $\mathrm{CT}$ radiomics. Eur Radiol. https://doi.org/10.1007/s00330-020-06872-z
28. Capobianco E, Dominietto M (2020) From medical imaging to radiomics: role of data science for advancing precision health. $\mathrm{J}$ Pers Med 10

29. Bogowicz M, Riesterer O, Ikenberg K et al (2017) Computed tomography radiomics predicts HPV status and local tumor control after definitive radiochemotherapy in head and neck squamous cell carcinoma. Int J Radiat Oncol Biol Phys 99:921-928

30. Zhong Y, Yuan M, Zhang T, Zhang YD, Li H, Yu TF (2018) Radiomics approach to prediction of occult mediastinal lymph node metastasis of lung adenocarcinoma. AJR Am J Roentgenol 211:109-113

31. Dou TH, Coroller TP, van Griethuysen JJM, Mak RH, Aerts H (2018) Peritumoral radiomics features predict distant metastasis in locally advanced NSCLC. PLoS One 13:e206108

32. Kim JY, Park JE, Jo Y et al (2019) Incorporating diffusion- and perfusion-weighted MRI into a radiomics model improves diagnostic performance for pseudoprogression in glioblastoma patients. Neuro Oncol 21:404-414

33. Suh HB, Choi YS, Bae S et al (2018) Primary central nervous system lymphoma and atypical glioblastoma: differentiation using radiomics approach. Eur Radiol 28:3832-3839

34. Li Y, Liu X, Xu K et al (2018) MRI features can predict EGFR expression in lower grade gliomas: a voxel-based radiomic analysis. Eur Radiol 28:356-362

35. Li Y, Qian Z, Xu K et al (2018) MRI features predict p53 status in lower-grade gliomas via a machine-learning approach. Neuroimage Clin 17:306-311

36. Wormald BW, Doran SJ, Ind TE, D'Arcy J, Petts J, deSouza NM (2020) Radiomic features of cervical cancer on T2-and diffusionweighted MRI: prognostic value in low-volume tumors suitable for trachelectomy. Gynecol Oncol 156:107-114

37. Cook GJ, Yip C, Siddique M et al (2013) Are pretreatment 18FFDG PET tumor textural features in non-small cell lung cancer associated with response and survival after chemoradiotherapy? J Nucl Med 54:19-26

38. Tixier F, Hatt M, Valla C et al (2014) Visual versus quantitative assessment of intratumor 18F-FDG PET uptake heterogeneity: prognostic value in non-small cell lung cancer. J Nucl Med 55: 1235-1241

39. Cook GJ, O'Brien ME, Siddique M et al (2015) Non-small cell lung cancer treated with erlotinib: heterogeneity of (18)F-FDG uptake at PET-association with treatment response and prognosis. Radiology 276:883-893

40. Parmar C, Leijenaar RT, Grossmann P et al (2015) Radiomic feature clusters and prognostic signatures specific for Lung and Head \& Neck cancer. Sci Rep 5:11044

41. Li S, Ding C, Zhang H, Song J, Wu L (2019) Radiomics for the prediction of EGFR mutation subtypes in non-small cell lung cancer. Med Phys 46:4545-4552

42. Mattonen SA, Davidzon GA, Benson J et al (2019) Bone marrow and tumor radiomics at (18)F-FDG PET/CT: impact on outcome prediction in non-small cell lung cancer. Radiology 293:451-459

43. Antunes J, Viswanath S, Rusu M et al (2016) Radiomics analysis on FLT-PET/MRI for characterization of early treatment response in renal cell carcinoma: a proof-of-concept study. Transl Oncol 9: $155-162$

44. Zamboglou C, Carles M, Fechter T et al (2019) Radiomic features from PSMA PET for non-invasive intraprostatic tumor discrimination and characterization in patients with intermediate- and high-risk prostate cancer - a comparison study with histology reference. Theranostics 9:2595-2605

45. Zheng X, Yao Z, Huang Y et al (2020) Deep learning radiomics can predict axillary lymph node status in early-stage breast cancer. Nat Commun 11:1236 
46. Caramella C, Allorant A, Orlhac F et al (2018) Can we trust the calculation of texture indices of CT images? A phantom study. Med Phys 45:1529-1536

47. Raunig DL, McShane LM, Pennello G et al (2015) Quantitative imaging biomarkers: a review of statistical methods for technical performance assessment. Stat Methods Med Res 24:27-67

48. Shaikh F, Franc B, Allen E et al (2018) Translational radiomics: defining the strategy pipeline and considerations for applicationpart 2: from clinical implementation to enterprise. J Am Coll Radiol 15:543-549

49. Shaikh F, Franc B, Allen E et al (2018) Translational radiomics: defining the strategy pipeline and considerations for applicationpart 1: from methodology to clinical implementation. J Am Coll Radiol 15:538-542

50. Zhao B, Tan Y, Tsai WY et al (2016) Reproducibility of radiomics for deciphering tumor phenotype with imaging. Sci Rep 6:23428

51. Pfaehler E, van Sluis J, Merema BBJ et al (2020) Experimental multicenter and multivendor evaluation of the performance of PET radiomic features using 3-dimensionally printed phantom inserts. J Nucl Med 61:469-476

52. Mackin D, Fave X, Zhang L et al (2017) Harmonizing the pixel size in retrospective computed tomography radiomics studies. PLoS One 12:e0178524

53. Nyul LG, Udupa JK, Zhang X (2000) New variants of a method of MRI scale standardization. IEEE Trans Med Imaging 19:143-150

54. Isaksson LJ, Raimondi S, Botta F et al (2020) Effects of MRI image normalization techniques in prostate cancer radiomics. Phys Med 71:7-13

55. Scalco E, Belfatto A, Mastropietro A et al (2020) T2w-MRI signal normalization affects radiomics features reproducibility. Med Phys 47:1680-1691

56. Leijenaar RT, Nalbantov G, Carvalho S et al (2015) The effect of SUV discretization in quantitative FDG-PET radiomics: the need for standardized methodology in tumor texture analysis. Sci Rep 5:11075

57. Lee SH, Cho HH, Lee HY, Park H (2019) Clinical impact of variability on $\mathrm{CT}$ radiomics and suggestions for suitable feature selection: a focus on lung cancer. Cancer Imaging 19:54

58. Duron L, Balvay D, Vande Perre S et al (2019) Gray-level discretization impacts reproducible MRI radiomics texture features. PLoS One 14:e0213459

59. Orlhac F, Frouin F, Nioche C, Ayache N, Buvat I (2019) Validation of a method to compensate multicenter effects affecting CT radiomics. Radiology 291:53-59

60. Rogers W, Thulasi Seetha S, Refaee TAG et al (2020) Radiomics: from qualitative to quantitative imaging. Br J Radiol 93:20190948

61. Zwanenburg A, Vallieres M, Abdalah MA et al (2020) The image biomarker standardization initiative: standardized quantitative radiomics for high-throughput image-based phenotyping. Radiology 295:328-338

62. Owens CA, Peterson CB, Tang C et al (2018) Lung tumor segmentation methods: impact on the uncertainty of radiomics features for non-small cell lung cancer. PLoS One 13:e0205003

63. Caballo M, Pangallo DR, Mann RM, Sechopoulos I (2020) Deep learning-based segmentation of breast masses in dedicated breast CT imaging: radiomic feature stability between radiologists and artificial intelligence. Comput Biol Med 118:103629

64. Hatt M, Lee JA, Schmidtlein CR et al (2017) Classification and evaluation strategies of auto-segmentation approaches for PET: report of AAPM task group No. 211. Med Phys 44:e1-e42

65. Waninger JJ, Green MD, Cheze Le Rest C, Rosen B, El Naqa I (2019) Integrating radiomics into clinical trial design. Q J Nucl Med Mol Imaging 63:339-346

66. Ciardo D, Jereczek-Fossa BA, Petralia G et al (2017) Multimodal image registration for the identification of dominant intraprostatic lesion in high-precision radiotherapy treatments. Br J Radiol 90: 20170021

67. Ou Y, Weinstein SP, Conant EF et al (2015) Deformable registration for quantifying longitudinal tumor changes during neoadjuvant chemotherapy. Magn Reson Med 73:2343-2356

68. Fornacon-Wood I, Mistry H, Ackermann CJ et al (2020) Reliability and prognostic value of radiomic features are highly dependent on choice of feature extraction platform. Eur Radiol 30: 6241-6250

69. Dhall DKR, Juneja M (2020) Machine learning: a review of the algorithms and its applications. In: Singh PKASY, Kolekar M, Tanwar S (eds) Proceedings of ICRIC 2019 Lecture Notes in Electrical Engineering. Springer, Cham

70. Ozgur C, Kleckner M, Li Y (2015) Selection of statistical software for solving big data problems: a guide for businesses, students, and universities. Sage Open 5:1-12

71. Pillai R, Oza P, Sharma P (2020) Review of machine learning techniques in health care. In: Singh P, Kar A, Singh Y, Kolekar M, Tanwar S (eds) Proceedings of ICRIC 2019 Lecture Notes in Electrical Engineering. Springer, Cham

72. Tanwani AK, Alfridi J, Shafiq Z, Farooq M (2009) Guidelines to select machine learning scheme for classification of biomedical datasets. In: Pizzuti C, Ritchie MD, Giacobini M (eds) Evolutionary Computation, Machine Learning and Data Mining in Bioinformatics EvoBIO 2009 Lecture Notes in Computer Science. Springer, Berlin, Heidelberg, pp 128-139

73. Chen T, Ning Z, Xu L et al (2019) Radiomics nomogram for predicting the malignant potential of gastrointestinal stromal tumours preoperatively. Eur Radiol 29:1074-1082

74. Parmar C, Grossmann P, Bussink J, Lambin P, Aerts H (2015) Machine Learning methods for quantitative radiomic biomarkers. Sci Rep 5:13087

75. Leger S, Zwanenburg A, Pilz K et al (2017) A comparative study of machine learning methods for time-to-event survival data for radiomics risk modelling. Sci Rep 7:13206

76. Afshar P, Mohammadi A, Plataniotis KN, Oikonomou A, Benali H (2019) From handcrafted to deep-learning-based cancer radiomics challenges and opportunities. Ieee Signal Processing Magazine 36:132-160

77. Vial A, Stirling D, Field M et al (2018) The role of deep learning and radiomic feature extraction in cancer-specific predictive modelling: a review. Transl Cancer Res 7:803-816

78. Avanzo M, Wei L, Stancanello J et al (2020) Machine and deep learning methods for radiomics. Med Phys 47:e185-e202

79. Peerlings J, Woodruff HC, Winfield JM et al (2019) Stability of radiomics features in apparent diffusion coefficient maps from a multi-centre test-retest trial. Sci Rep 9:4800

80. Traverso A, Wee L, Dekker A, Gillies R (2018) Repeatability and reproducibility of radiomic features: a systematic review. Int J Radiat Oncol Biol Phys 102:1143-1158

81. AlBadawy EA, Saha A, Mazurowski MA (2018) Deep learning for segmentation of brain tumors: impact of cross-institutional training and testing. Med Phys 45:1150-1158

82. Larue R, Klaassen R, Jochems A et al (2018) Pre-treatment CT radiomics to predict 3-year overall survival following chemoradiotherapy of esophageal cancer. Acta Oncol 57:1475-1481

83. Soufi M, Arimura H, Nagami N (2018) Identification of optimal mother wavelets in survival prediction of lung cancer patients using wavelet decomposition-based radiomic features. Med Phys 45:5116-5128

84. Xu X, Huang L, Chen J et al (2019) Application of radiomics signature captured from pretreatment thoracic CT to predict brain metastases in stage III/IV ALK-positive non-small cell lung cancer patients. J Thorac Dis 11:4516-4528

85. Li H, Xie Y, Wang X, Chen F, Sun J, Jiang X (2019) Radiomics features on non-contrast computed tomography predict early 
enlargement of spontaneous intracerebral hemorrhage. Clin Neurol Neurosurg 185:105491

86. Huynh E, Coroller TP, Narayan V et al (2016) CT-based radiomic analysis of stereotactic body radiation therapy patients with lung cancer. Radiother Oncol 120:258-266

87. Hui B, Qiu JJ, Liu JH, Ke NW (2020) Identification of pancreaticoduodenectomy resection for pancreatic head adenocarcinoma: a preliminary study of radiomics. Comput Math Methods Med 2020:2761627

88. Leithner D, Horvat JV, Marino MA et al (2019) Radiomic signatures with contrast-enhanced magnetic resonance imaging for the assessment of breast cancer receptor status and molecular subtypes: initial results. Breast Cancer Res 21:106

89. Zhang Y, Yan P, Liang F, Ma C, Liang S, Jiang C (2019) Predictors of epilepsy presentation in unruptured brain arteriovenous malformations: a quantitative evaluation of location and radiomics features on T2-weighted imaging. World Neurosurg 125:e1008-e1015

90. Zhou J, Lu J, Gao C et al (2020) Predicting the response to neoadjuvant chemotherapy for breast cancer: wavelet transforming radiomics in MRI. BMC Cancer 20:100

91. Lue KH, Wu YF, Liu SH et al (2019) Intratumor heterogeneity assessed by (18)F-FDG PET/CT predicts treatment response and survival outcomes in patients with Hodgkin lymphoma. Acad Radiol. https://doi.org/10.1016/j.acra.2019.10.015

92. Shiri I, Maleki H, Hajianfar G et al (2020) Next-generation radiogenomics sequencing for prediction of EGFR and KRAS mutation status in NSCLC patients using multimodal imaging and machine learning algorithms. Mol Imaging Biol. https://doi. org/10.1007/s11307-020-01487-8

93. Lee SH, Han P, Hales R et al (2020) Multi-view radiomics and dosiomics analysis with machine learning for predicting acutephase weight loss in lung cancer patients treated with radiotherapy. Phys Med Biol. https://doi.org/10.1088/1361-6560/ab8531

94. Nazari M, Shiri I, Hajianfar G et al (2020) Noninvasive Fuhrman grading of clear cell renal cell carcinoma using computed tomography radiomic features and machine learning. Radiol Med. https://doi.org/10.1007/s11547-020-01169-z

95. Bhatia A, Birger M, Veeraraghavan $\mathrm{H}$ et al (2019) MRI radiomic features are associated with survival in melanoma brain metastases treated with immune checkpoint inhibitors. Neuro Oncol 21: $1578-1586$

96. Shayesteh SP, Alikhassi A, Fard Esfahani A et al (2019) Neoadjuvant chemoradiotherapy response prediction using MRI based ensemble learning method in rectal cancer patients. Phys Med 62: 111-119

97. Fiset S, Welch ML, Weiss J et al (2019) Repeatability and reproducibility of MRI-based radiomic features in cervical cancer. Radiother Oncol 135:107-114

98. Fave X, Zhang L, Yang J et al (2017) Delta-radiomics features for the prediction of patient outcomes in non-small cell lung cancer. Sci Rep 7:588
99. Dong X, Sun X, Sun L et al (2016) Early change in metabolic tumor heterogeneity during chemoradiotherapy and its prognostic value for patients with locally advanced non-small cell lung cancer. PLoS One 11:e157836

100. Tixier F, Vriens D, Cheze-Le Rest C et al (2016) Comparison of tumor uptake heterogeneity characterization between static and parametric 18F-FDG PET images in non-small cell lung cancer. J Nucl Med 57:1033-1039

101. Yin P, Mao N, Zhao C, Wu J, Chen L, Hong N (2019) A tripleclassification radiomics model for the differentiation of primary chordoma, giant cell tumor, and metastatic tumor of sacrum based on T2-weighted and contrast-enhanced T1-weighted MRI. J Magn Reson Imaging 49:752-759

102. Grossmann P, Stringfield O, El-Hachem N et al (2017) Defining the biological basis of radiomic phenotypes in lung cancer. Elife 6

103. Tu W, Sun G, Fan L et al (2019) Radiomics signature: a potential and incremental predictor for EGFR mutation status in NSCLC patients, comparison with CT morphology. Lung Cancer 132:2835

104. Sanghani P, Ang BT, King NKK, Ren H (2018) Overall survival prediction in glioblastoma multiforme patients from volumetric, shape and texture features using machine learning. Surg Oncol 27: 709-714

105. Ferreira Junior JR, Koenigkam-Santos M, Cipriano FEG, Fabro AT, Azevedo-Marques PM (2018) Radiomics-based features for pattern recognition of lung cancer histopathology and metastases. Comput Methods Programs Biomed 159:23-30

106. Penzias G, Singanamalli A, Elliott R et al (2018) Identifying the morphologic basis for radiomic features in distinguishing different Gleason grades of prostate cancer on MRI: preliminary findings. PLoS One 13:e0200730

107. Holm EA (2019) In defense of the black box. Science 364:26-27

108. Oakden-Rayner L, Carneiro G, Bessen T, Nascimento JC, Bradley AP, Palmer LJ (2017) Precision radiology: predicting longevity using feature engineering and deep learning methods in a radiomics framework. Sci Rep 7:1648

109. Bibault JE, Giraud P, Housset M et al (2018) Deep learning and radiomics predict complete response after neo-adjuvant chemoradiation for locally advanced rectal cancer. Sci Rep 8:12611

110. Ning Z, Luo J, Li Y et al (2019) Pattern classification for gastrointestinal stromal tumors by integration of radiomics and deep convolutional features. IEEE J Biomed Health Inform 23:11811191

111. Shboul ZA, Alam M, Vidyaratne L, Pei L, Elbakary MI, Iftekharuddin KM (2019) Feature-guided deep radiomics for glioblastoma patient survival prediction. Front Neurosci 13:966

Publisher's note Springer Nature remains neutral with regard to jurisdictional claims in published maps and institutional affiliations. 


\section{Affiliations}

Laure Fournier $^{1,2,3} \cdot$ Lena Costaridou $^{2,4}$ • Luc Bidaut ${ }^{3,5}$ - Nicolas Michoux ${ }^{3,6}$. Frederic E. Lecouvet ${ }^{3,6}$. Lioe-Fee de Geus-Oei ${ }^{3,7,8} \cdot$ Ronald Boellaard $^{2,9,10}$ - Daniela E. Oprea-Lager ${ }^{3,9}$. Nancy A Obuchowski ${ }^{10,11}$. Anna Caroli ${ }^{2,12}$ • Wolfgang G. Kunz ${ }^{3,13}$. Edwin H. Oei ${ }^{2,14}$ • James P. B. O'Connor ${ }^{2,15}$ - Marius E. Mayerhoefer ${ }^{2,16}$. Manuela Franca ${ }^{2,17}$. Angel Alberich-Bayarri ${ }^{2,18}$. Christophe M. Deroose $3,19,20$. Christian Loewe . $^{2,21}$. Rashindra Manniesing ${ }^{2,22}$ - Caroline Caramella ${ }^{3,23} \cdot$ Egesta Lopci ${ }^{3,24}$ - Nathalie Lassau 2,3,10,25 . Anders Persson ${ }^{2,26}$. Rik Achten $^{2,27}$ - Karen Rosendahl ${ }^{2,28}$. Olivier Clement ${ }^{1,2}$ - Elmar Kotter ${ }^{2,29}$ - Xavier Golay ${ }^{2,10,30}$ - Marion Smits ${ }^{2,3,14}$. Marc Dewey $^{2,31}$ - Daniel C. Sullivan 2,10,32 - Aad van der Lugt ${ }^{2,14}$ - Nandita M. deSouza 2,3,10,33 (D) European Society of Radiology ${ }^{34}$

1 PARCC, INSERM, Radiology Department, AP-HP, Hopital europeen Georges Pompidou, Université de Paris, F-

75015 Paris, France

2 European Imaging Biomarkers Alliance (EIBALL), European Society of Radiology, Vienna, Austria

3 Imaging Group, European Organisation of Research and Treatment in Cancer (EORTC), Brussels, Belgium

4 School of Medicine, University of Patras, University Campus, Rio, 26500 Patras, Greece

5 College of Science, University of Lincoln, Lincoln LN6 7TS, UK

6 Department of Radiology, Institut de Recherche Expérimentale et Clinique (IREC), Cliniques Universitaires Saint Luc, Université Catholique de Louvain (UCLouvain), B-1200 Brussels, Belgium

7 Department of Radiology, Leiden University Medical Center, Leiden, The Netherlands

8 Biomedical Photonic Imaging Group, University of Twente, Enschede, The Netherlands

9 Department of Radiology \& Nuclear Medicine, Cancer Centre Amsterdam, Amsterdam University Medical Centers (VU University), Amsterdam, The Netherlands

10 Quantitative Imaging Biomarkers Alliance, Radiological Society of North America, Oak Brook, IL, USA

11 Department of Quantitative Health Sciences, Cleveland Clinic, Cleveland, OH, USA

12 Department of Biomedical Engineering, Istituto di Ricerche Farmacologiche Mario Negri IRCCS, Bergamo, Italy

13 Department of Radiology, University Hospital, LMU Munich, Munich, Germany

14 Department of Radiology \& Nuclear Medicine, Erasmus MC, University Medical Center, Rotterdam, The Netherlands

15 Division of Cancer Sciences, University of Manchester, Manchester, UK

16 Department of Biomedical Imaging and Image-guided Therapy, Medical University of Vienna, Vienna, Austria

17 Department of Radiology, Centro Hospitalar Universitário do Porto, Instituto de Ciências Biomédicas de Abel Salazar, University of Porto, Porto, Portugal
18 Quantitative Imaging Biomarkers in Medicine (QUIBIM), Valencia, Spain

19 Nuclear Medicine, University Hospitals Leuven, Leuven, Belgium

20 Nuclear Medicine and Molecular Imaging, Department of Imaging and Pathology, KU Leuven, Leuven, Belgium

21 Division of Cardiovascular and Interventional Radiology, Dept. for Bioimaging and Image-Guided Therapy, Medical University of Vienna, Vienna, Austria

22 Department of Radiology and Nuclear Medicine, Radboud University Medical Center, 6525 GA Nijmegen, The Netherlands

23 Radiology Department, Hôpital Marie Lannelongue, Institut d'Oncologie Thoracique, Université Paris-Saclay, Le PlessisRobinson, France

24 Nuclear Medicine, Humanitas Clinical and Research Hospital IRCCS, Rozzano, MI, Italy

25 Imaging Department, Gustave Roussy Cancer Campus Grand, Paris, UMR 1281, INSERM, CNRS, CEA, Universite Paris-Saclay, Saint-Aubin, France

26 Department of Radiology, and Department of Health, Medicine and Caring Sciences, Center for Medical Image Science and Visualization (CMIV), Linköping University, Linköping, Sweden

27 Department of Radiology and Medical Imaging, Ghent University Hospital, Gent, Belgium

28 Department of Radiology, University Hospital of North Norway, Tromsø, Norway

29 Department of Radiology, University Medical Center Freiburg, Freiburg, Germany

30 Queen Square Institute of Neurology, University College London, London, UK

31 Department of Radiology, Charité Universitätsmedizin Berlin, Berlin, Germany

32 Dept. of Radiology, Duke University, 311 Research Dr, Durham, NC 27710, USA

33 Division of Radiotherapy and Imaging, The Institute of Cancer Research and Royal Marsden NHS Foundation Trust, London, UK

34 Am Gestade 1, 1010 Vienna, Austria 